\title{
Compound heterozygous hemophilia $A$ in a female patient and the identification of a novel missense mutation, p.Met1093Ile
}

\author{
SHU-KAI QIAO ${ }^{1,2}$, HAN-YUN REN $^{1}$, JIN-HAI REN $^{2}$ and XIAO-NAN GUO ${ }^{2}$ \\ ${ }^{1}$ Department of Hematology, Peking University First Hospital, Beijing 100034; ${ }^{2}$ Department of Hematology, \\ The Second Hospital of Hebei Medical University, Shijiazhuang, Hebei 050000, P.R. China
}

Received June 18, 2013; Accepted November 25, 2013

DOI: $10.3892 / \mathrm{mmr} .2013 .1841$

\begin{abstract}
Hemophilia A (HA) in females is rare. Female HA cases are often misdiagnosed as acquired HA (AHA) or as von Willebrand disease type $2 \mathrm{~N}$ (vWD-2N). Here, we report the case of a 37-year-old female HA patient with a moderate factor VIII (FVIII) deficiency. The patient had no personal or family history of bleeding disorders, but presented with heavy uterine bleeding following surgery to remove an intrauterine device. IgG inhibitory antibodies against FVIII were undetected. A compound heterozygote mutation of the FVIII gene (F8) was found in this patient. The p.Val502Asp mutation, which has been reported previously, affects A2 domain function. A novel missense point mutation, p.Met1093Ile, was identified in the B domain. The compound heterozygote mutations in F8, p.Val502Asp and p.Met1093Ile, caused HA in this female patient, with a moderate phenotype.
\end{abstract}

\section{Introduction}

Hemophilia A (HA) is an X-linked recessive congenital coagulation disorder caused by a factor VIII (FVIII) deficiency, which results from mutations in the FVIII gene (F8). HA typically occurs in males with an incidence of $\sim 1$ in 5000 (1). Female HA patients have rarely been reported, although they may carry a defective allele. Human F8 maps to the long arm of the Xq28 region of the X chromosome and consists of 26 exons and 25 introns. The protein product of $\mathrm{F} 8$ possesses no enzyme activity. The 19 amino acid signal peptide at the $\mathrm{N}$-terminus, encoded by an open reading frame (ORF), leads to the passage of FVIII through hepatocytes to blood vessels (2). The matured FVIII protein is composed of 2332 amino acids and comprises three homologous A domains, two homologous $\mathrm{C}$ domains and a single $\mathrm{B}$ domain. These domains, arranged

Correspondence to: Professor Han-Yun Ren, Department of Hematology, Peking University First Hospital, 8 Xishiku Street, Xicheng, Beijing 100034, P.R. China

E-mail: renhy0813@163.com

Key words: factor VIII, hemophilia A, female, F8, mutation as $\mathrm{A} 1-\mathrm{A} 2-\mathrm{B}-\mathrm{A} 3-\mathrm{C} 1-\mathrm{C} 2$ from the $\mathrm{N}$ - to the $\mathrm{C}$-terminus, are important for FVIII function. FVIII synthesized in hepatocytes is secreted into the plasma in the form of an inactive pro-cofactor (3). FVIII, an essential cofactor for coagulation factor IX, readily binds to von Willebrand factor (vWF), forming a tight non-covalent complex that protects against degradation. Furthermore, FVIII may also interact with a number of proteins, such as thrombin and factor X. These interactions are indispensable for effective coagulation. Once FVIII is cleaved by thrombin, activated FVIII dissociates from vWF and participates in the coagulation cascade (4).

Genetic abnormalities in F8 may result in qualitative or quantitative defects of the FVIII protein. The most common F8 abnormality to cause HA is an inversion of intron 1 or $22(5,6)$. The remaining HA cases are caused by numerous point mutations spread throughout the gene, including missense, nonsense, splice site, frameshift mutations and gross deletions $(7,8)$. To date, $>1400 \mathrm{~F} 8$ mutations within the FVIII coding and non-coding regions have been identified in the Haemophilia A Mutation, Search, Test and Resource Site (HAMSTeRS) database (http://hadb.org.uk/).

In this study, we report the case of a female HA patient with F8 compound heterozygote mutations. Diagnosis and differential diagnosis were complex. Furthermore, F8 compound heterozygote mutations (c.1505T >A, p.Va1502Asp and c.3279G $>$ A, p.Met1093Ile) were identified using multiplex PCR and DNA sequencing.

\section{Case report}

Patient history. A 37-year-old female patient presented with an 8-month history of severe uterine abnormal bleeding following surgery for the removal of an intrauterine device. After a short time, the patient developed profound anemia (hemoglobin level of $58 \mathrm{~g} / \mathrm{l}$ ). Prior to being admitted to our hospital, the patient had received a blood transfusion $(1200 \mathrm{ml})$ for hemorrhagic anemia in the Second Hospital of Hebei Medical University. Furthermore, the patient complained of repeated skin purpura since childhood. The patient had no other history of bleeding, such as nosebleeds, gingival bleeding, hematemesis or melena. There was no personal or family history of bleeding disorders. The patient denied any intake of medicines or herbs. The biochemical and hematological data of the patient are shown in Table I. 
Table I. Initial biochemistry and hematology results.

\begin{tabular}{lcc}
\hline Variable & Value & Reference range \\
\hline Whole blood & & \\
White blood cell & $5.8 \times 10^{9} / 1$ & $(4-10) \times 10^{9} / 1$ \\
Hemoglobin & $92 \mathrm{~g} / \mathrm{l}$ & $110-155 \mathrm{~g} / 1$ \\
Platelet & $216 \times 10^{9} / 1$ & $(100-300) \times 10^{9} / 1$ \\
Coagulation panel & & \\
PT & $14.6 \mathrm{sec}$ & $12-16 \mathrm{sec}$ \\
INR & 1.12 & $0.8-1.2$ \\
APTT & $46.5 \mathrm{sec}$ & $24-37 \mathrm{sec}$ \\
APTT-R & 1.62 & $0.8-1.2$ \\
Fib & $242 \mathrm{mg} / \mathrm{dl}$ & $200-400 \mathrm{mg} / \mathrm{dl}$ \\
BT & $11 \mathrm{~min}$ & $4.8-9 \mathrm{~min}$ \\
FVIII:C & $4.7 \%$ & $50-150 \%$ \\
vWF-Ag & $128 \%$ & $60-150 \%$ \\
LA & $29.5 \mathrm{sec}$ & $27-41 \mathrm{sec}$ \\
RIPA (1.2 mg/ml) & $91 \%$ & $87-102 \%$ \\
Factor VIII antibody & Negative & Negative \\
\hline
\end{tabular}

PT, prothrombin time; INR, international normalized ratio; APTT: activated partial thromboplastin time; Fib, fibrinogen; BT, bleeding time; vWF-Ag, von Willebrand factor antigen; LA, lupus anticoagulant; RIPA, ristocetin induced platelet agglutination.

White blood cell and platelet levels were within the normal ranges. Hemoglobin was only marginally reduced (92 g/l), due to the blood transfusion. The prothrombin time (PT) and international normalized ratio (INR) were normal, but the activated partial thromboplastin time (APTT) and bleeding time (BT) were noticeably prolonged, which may be corrected easily by a plasma transfusion. Plasma FVIII levels were significantly decreased (4.7\%). vWF antigen levels were within the normal range (128\%). Autoimmune markers, such as the antinuclear, anticardiolipin, antidouble stranded DNA and extractable nuclear antigen (ENA) polypeptide antibodies were all negative. Other coagulation tests were also normal, including platelet count and function, and fibrinogen levels.

Diagnosis and differential diagnosis. Based on the clinical manifestations and laboratory tests, the following diagnoses were considered, in order of decreasing probability: i) Acquired HA (AHA); ii) von Willebrand disease type 2N (vWD-Type $2 \mathrm{~N}$ ); and iii) HA. There is a certain degree of overlap in the clinical manifestations and laboratory results among these three disorders. Although female HA is extremely rare, it could not be ruled out as a possibility. The key points of diagnosis and differential diagnosis for these three diseases are discussed in the following paragraphs.

AHA is an autoimmune disorder caused by autoantibodies against FVIII, neutralizing its coagulation functions and resulting in severe, often life-threatening bleeding. It is characterized by severe, spontaneous hemorrhaging at sites, such as the skin, muscle and soft tissues, or by excessive bleeding during surgery. Hemarthrosis, the hallmark of severe congenital HA, seldom occurs in AHA patients (9). AHA may be associated with several clinical conditions, including
Table II. Differential diagnosis of HA, vWD-Type 2N and AHA.

\begin{tabular}{lccc}
\hline Variable & HA & vWD-Type 2N & AHA \\
\hline BT & Normal & Normal or $(\uparrow)$ & Normal \\
APTT & $\uparrow$ & $\uparrow$ & $\uparrow$ \\
FVIII:C & $\downarrow$ & $\downarrow$ & $\downarrow$ \\
vWF-Ag & Normal & $(\downarrow)$ or Normal & Normal \\
FVIII inhibitor & Negative & Negative & Positive \\
vWF-FVIII & Normal & $\downarrow$ & Normal \\
binding affinity & & & \\
vWF:Rcof & Normal & $(\downarrow)$ or Normal & Normal \\
\hline
\end{tabular}

HA: hemophilia A; AHA: Acquired HA; vWD, von Willebrand disease; FVIII:C, coagulation factor VIII activity; vWF, von Willebrand factor; vWF:Ag, vWF antigen; vWF:RCof, vWF ristocetin cofactor activity.

pregnancy, autoimmune diseases, malignancies, infections or drugs. Approximately $50 \%$ of patients are idiopathic with no known underlying disease association (10). If a patient presents with spontaneous hemorrhaging, then previous personal or family history of bleeding must be ruled out. Once laboratory results reveal the isolated prolongation of APTT and reduced FVIII levels, which fail to normalize following a normal plasma transfusion, AHA should be suspected. Following confirmation that FVIII antibodies are absent and that FVIII levels may be improved by transfusing normal plasma, a diagnosis of AHA was definitively ruled out.

vWD is the most common inherited bleeding disorder and is caused by deficiency or dysfunction of vWF. vWD is divided into three types: Type 1 (partial quantitative deficiency); Type 2, with four subtypes $2 \mathrm{~A}, 2 \mathrm{~B}, 2 \mathrm{M}$ and $2 \mathrm{~N}$ (qualitative deficiency); and Type 3 (complete quantitative deficiency) (11). Type $2 \mathrm{~N}$ vWD show a marked decrease in the vWF binding affinity for FVIII. Its clinical manifestations are similar to that of mild HA: FVIII levels are decreased, but vWF antigen (vWF:Ag) levels are within the normal range (12). These patients are usually misdiagnosed as HA. When the laboratory results were returned, it was observed that the vWF binding affinity for FVIII and the ristocetin-induced platelet agglutination (RIPA) were normal in our patient. Therefore, a diagnosis of vWD-Type $2 \mathrm{~N}$ was ruled out. Thus, a diagnosis of HA was considered for the patient. The differential diagnoses of HA, VWD-Type $2 \mathrm{~N}$ and AHA are outlined in Table II.

Mutational screening. In order to confirm the cause of HA in the patient, a mutational screening of F8 was performed. Genomic DNA was obtained from EDTA-anticoagulated blood from the patient. Inversions in introns 1 and 22 were first screened using long-distance PCR (13). All exons and their flanking regions of F8 were amplified by PCR using specific primers, as described previously (14). PCR products were purified and sequenced.

DNA sequencing. Introns 1 and 22 were not inverted. A previously reported mutation (15) was detected (exon 10, 
A

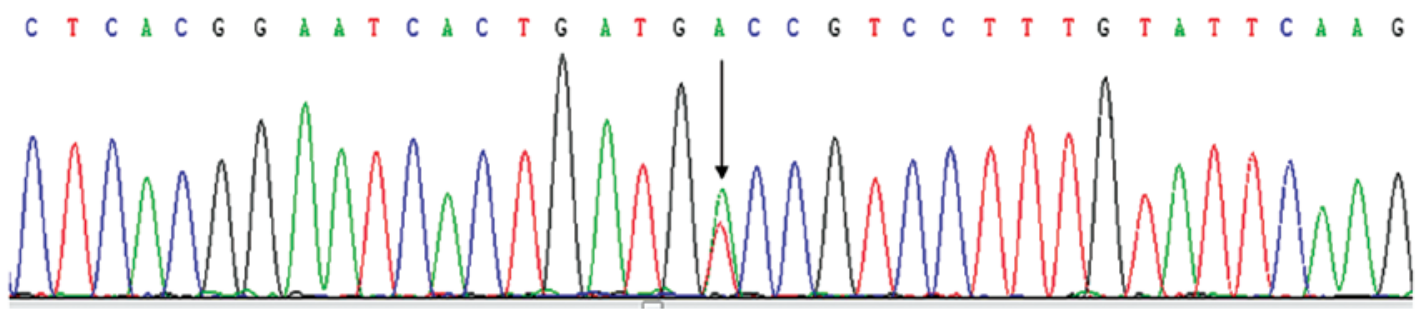

B

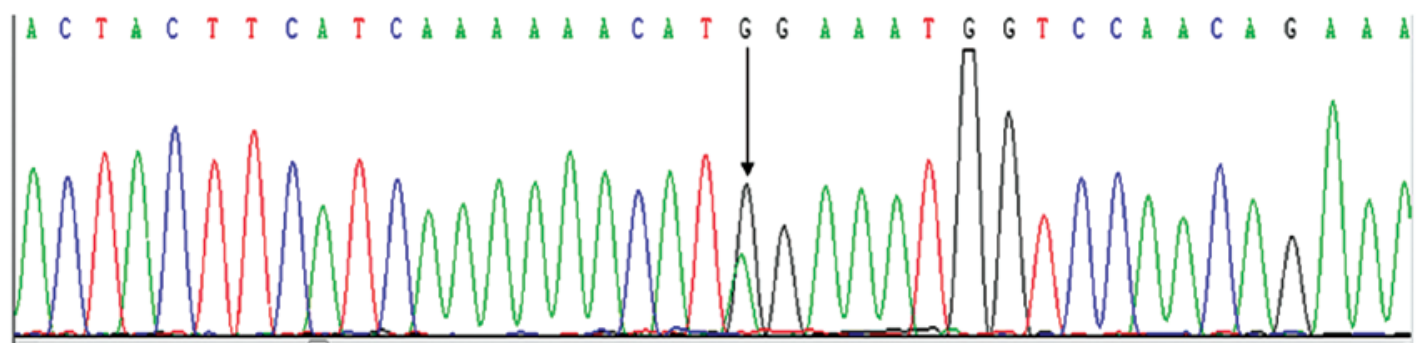

Figure 1. Results from DNA sequencing of exons 10 and 14. (A) Exon 10: NM_000132.3 c.1505T>A (p.Val502Asp). (B) Exon 14: NM_000132.3 c.3279G>A (p.Met1093Ile).

A

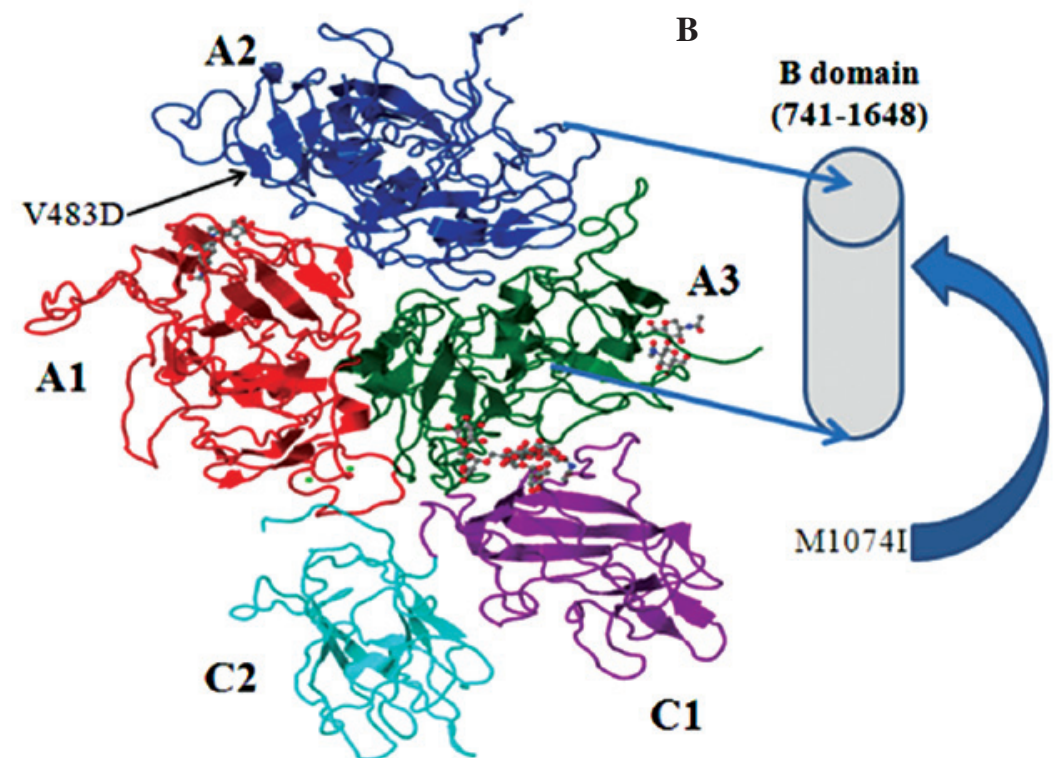

C
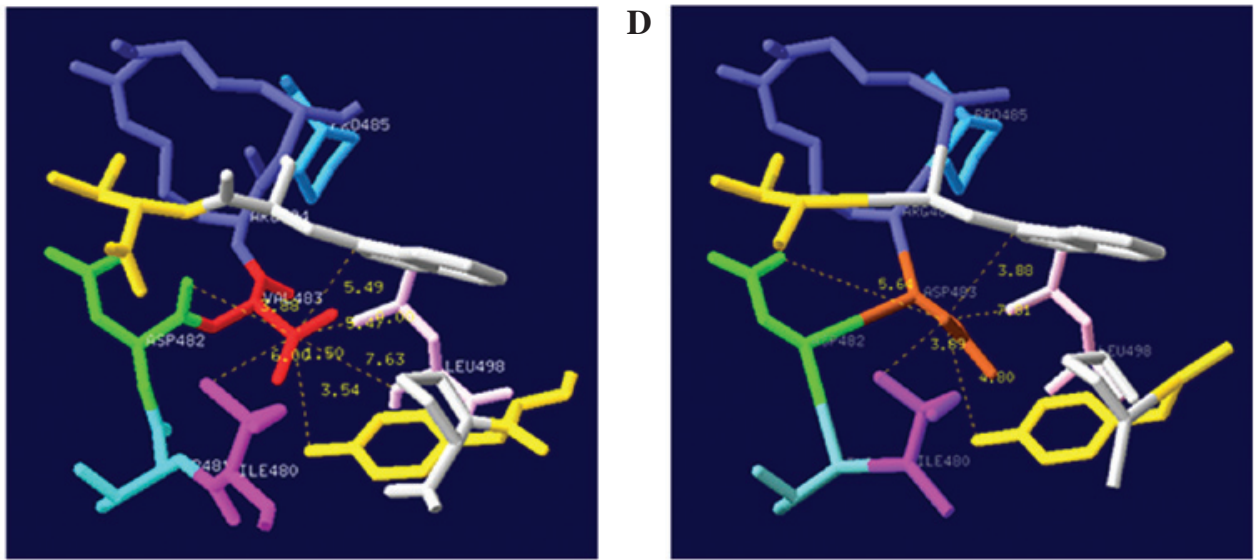

Figure 2. Modeled structure of compound heterozygote mutated FVIII protein. (A) Crystal structure of B domain-deleted FVIII (PDB ID 2R7E). The domains are individually labeled and colored. The p.Val502Asp mutation located in the A2 domain is indicated by an arrow. (B) Simple model of the FVIII B domain. The p.Met1093Ile mutation is located in the B domain and is indicated by an arrow. The B domain spans the sequence from amino acid 741 to 1648 . Unlike A and C domains, no available molecular models or crystal structures helped to elucidate the detailed structure of the B domain. (C and D) Comparison of p.Val502Asp mutation structural images in A2 domain of FVIII generated using the Swiss-Pdb Viewer. 


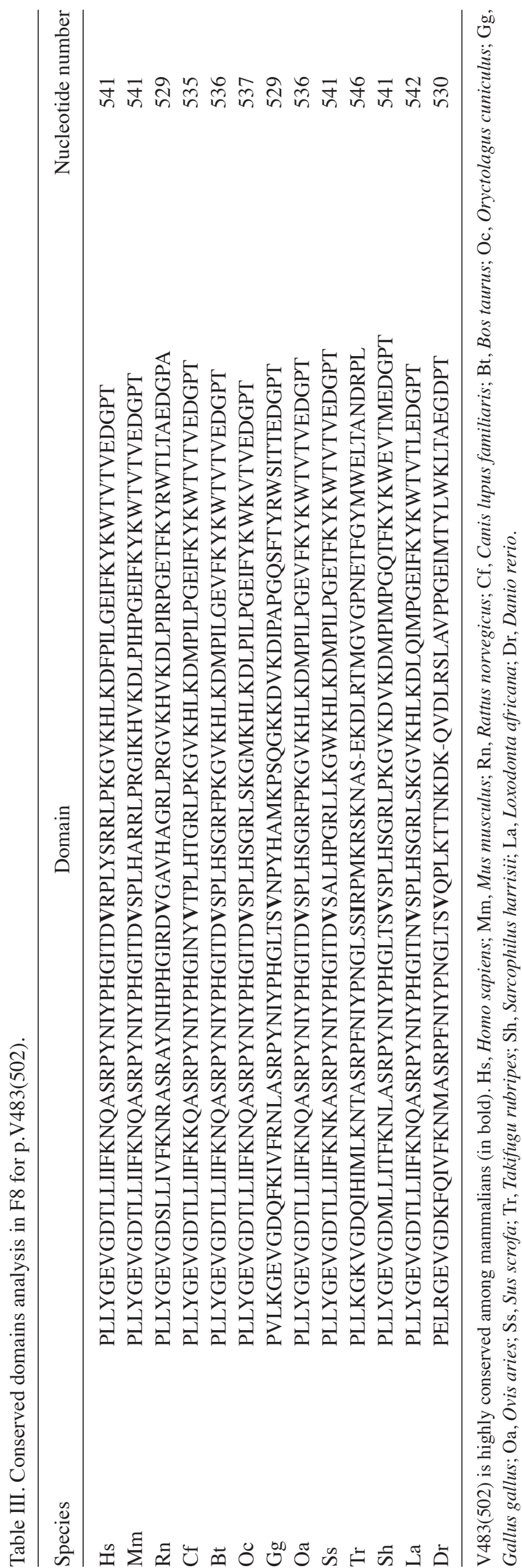

c.1505T $>$ A, p.Val502Asp), and a novel unreported missense point mutation (exon 14, c.3279G>A, p.Met1093Ile) (Fig. 1).

Molecular modeling. Mutated FVIII proteins were modeled using the crystal structure of activated recombinant FVIII $(16,17)$. Structural images were generated using the Swiss-Pdb Viewer (Swiss Institute of Bioinformatics, Geneva, Switzerland) (18) (Fig. 2).

Conserved domains analysis. A conserved domains analysis was conducted for p.Val502 and p.Met1093 by using the Multiple Alignment Tool (http://www.ncbi.nlm.nih.gov/tools/ cobalt/cobalt.cgi?link_loc=BlastHomeLink). It was observed that p.Val502 of the A2 domains in F8 is highly conserved among mammals (Table III). By contrast, p.Met1093 in the B domain shares little amino acid homology, indicating that it is not conserved among different species.

\section{Discussion}

Due to the X-linked recessive mode of inheritance, HA usually affects males, and females usually are carriers who may pass the disease on to their progeny (19). Thus, female HA cases are rarely observed. However, in certain cases, there are a variety of potential genetic mechanisms leading to HA in females: i) Non-random inactivation of the normal $\mathrm{X}$-chromosome in a female HA carriers (the most common cause of female HA $(20,21)$; ii) homozygous F8 mutations (mostly reported in India where consanguineous marriages are more common) $(22,23)$; iii) compound heterozygous mutations affecting both F8 alleles (21); and iv) X-chromosome monosomy or gross structural defects, such as a deletion or translocation (24-26).

Disease severity in HA patients is classified according to residual plasma FVIII activity (FVIII:C): severe $(<1 \%)$, moderate (1-5\%) and mild (5-35\%) (27). In the severe phenotype, the most prevalent mutations are inversions of introns 1 or 22 , accounting for $5 \%$ and $40-50 \%$ of patients, respectively $(5,28)$. Moderate or mild HA are usually caused by missense mutations (29).

In this study, we presented the case of a female HA patient with a moderately clinical phenotype (FVIII: $\mathrm{C}=4.8 \%$ ) resulting from $\mathrm{F} 8$ compound heterozygous mutations. The p.Val502Asp mutation located in A2 domains was first reported to be associated with mild HA by Fernández-López et al (15) in 2005. As the original amino acid Val502 in F8 is highly conserved among all known mammals, it was predicted that Val502 is involved in an A2-specific functional role. The non-polar, hydrophobic amino acid Val is replaced by the polar and acidic amino acid Asp, resulting in altered stability and protein folding. The p.Met1093Ile mutation is located in the B domain of F8, which is encoded by exon 14 (amino acids 741-1648). Contrary to the $\mathrm{A}$ and $\mathrm{C}$ domains, there are no available molecular models or crystal structures that elucidate the detailed structural information of the B domain. The FVIII B domain shares little sequence homology with other known mammalian species. Despite the fact that the B domain is not directly required for FVIII coagulation activity, it has been shown to exhibit a major role in the intracellular interactions that regulate quality control and secretion, as well as 
potential regulatory roles within plasma during activation, platelet binding, inactivation and clearance (30). Therefore, the p.Met1093Ile mutation in the B domain also affects FVIII coagulation activity. Furthermore, missense mutations within the F8 B domain have often been reported in HA patients $(31,32)$. p.Met1093Ile is a novel missense mutation unreported in the HAMSTeRS database. The mutation was submitted to the HAMSTeRS database and was accepted (unpublished). It was estimated that the compound heterozygous mutations (p.Val502Asp and p.Met1093Ile) are causative gene defects, which resulted in a moderate HA phenotype in this female patient.

In conclusion, female HA patients, particularly those without a personal or family history of bleeding disorders, are often misdiagnosed as AHA or vWD-type $2 \mathrm{~N}$. A diagnosis of HA should be made in a female patient when isolated APTT is prolonged, FVIII binding capacity for vWF is normal, FVIII levels are decreased and FVIII auto-antibodies are absent. Genetic analysis of F8 using multiplex PCR and DNA sequencing is an essential tool in elucidating the nature of the various molecular mechanisms resulting in HA in females.

\section{Acknowledgements}

The authors are grateful to Dr Ye-ling Lu (Ruijin Hospital, Shanghai Jiaotong University School of Medicine, Shanghai, China) for his technical assistance in mutational analysis and to Professor Jing-yu Zhang (The Second Hospital of Hebei Medical University, Shijiazhuang, Hebei, China) for measuring the vWF binding affinity for FVIII.

\section{References}

1. Klinge J, Ananyeva NM, Hauser CA and Saenko EL: Hemophilia A - from basic science to clinical practice. Semin Thromb Hemost 28: 309-322, 2002.

2. Saenko EL, Ananyeva NM, Tuddenham EG and Kemball-Cook G: Factor VIII - novel insights into form and function. Br J Haematol 119: 323-331, 2002.

3. Lenting PJ, van Mourik JA and Mertens K: The life cycle of coagulation factor VIII in view of its structure and function. Blood 92: 3983-3996, 1998.

4. Lenting PJ, Pegon JN, Christophe OD and Denis CV: Factor VIII and von Willebrand factor - too sweet for their own good. Haemophilia 16: 194-199, 2010.

5. Bagnall RD, Waseem N, Green PM and Giannelli F: Recurrent inversion breaking inversion intron 1 of the factor VIII gene is a frequent cause of severe hemophilia A. Blood 99: 168-174, 2002.

6. Oldenburg J, Brackmann HH, Hanfland P and Schwaab R: Molecular genetics in haemophilia A. Vox Sang 78: 33-38, 2000

7. Castaldo G, D'Argenio V, Nardiello P, et al: Haemophilia A: molecular insights. Clin Chem Lab Med 45: 450-461, 2007.

8. Oldenburg J: Mutation profiling in haemophilia A. Thromb Haemost 85: 577-579, 2001

9. Shetty S, Bhave M and Ghosh K: Acquired hemophilia A: diagnosis, aetiology, clinical spectrum and treatment options. Autoimmun Rev 10: 311-316, 2011.

10. Franchini M, Targher G, Montagnana M and Lippi G: Laboratory, clinical and therapeutic aspects of acquired hemophilia A. Clin Chim Acta 395: 14-18, 2008.

11. Sadler JE, Budde U, Eikenboom JC, et al: Update on the pathophysiology and classification of von Willebrand disease: A report of the Subcommittee on von Willebrand Factor. J Thromb Haemost 4: 2103-2114, 2006.
12. Nichols WL, Rick ME, Ortel TL, et al: Clinical and laboratory diagnosis of von Willebrand disease: a synopsis of the 2008 NHLBI/NIH guidelines. Am J Hematol 84: 366-370, 2009.

13. Keeney S, Mitchell M, Goodeve A and the UK Haemophilia Center Doctors' Organisation Harmophilia Genetics Laboratory Network: The molecular analysis of haemophilia A: a guideline from the UK haemophilia centre doctors' organization haemophilia genetics laboratory network. Haemophilia 11: 387-397, 2005.

14. Boekhorst J, Verbruggen B, Lavergne JM, et al: Thirteen novel mutations in the factor VIII gene in the Nijmegen haemophilia A patient population. Br J Haematol 131: 109-117, 2005.

15. Fernández-López O, García-Lozano JR, Núñez-Vázquez R, Pérez-Garrido R and Núñez-Roldán A: The spectrum of mutations in Southern Spanish patients with hemophilia A and identification of 28 novel mutations. Haematologica 90: 707-710, 2005.

16. Shen BW, Spiegel PC, Chang CH, et al: The tertiary structure and domain organization of coagulation factor VIII. Blood 111: 1240-1247, 2008.

17. Kopp J and Schwede T: The SWISS-MODEL repository of annotated 3-dimensional protein structure homology models. Nucleic Acids Res 32: D230-D234, 2004.

18. Guex N and Peitsch MC: SWISS-MODEL and the Swiss-PdbViewer: an environment for comparative protein modeling. Electrophoresis 18: 2714-2723. 1997.

19. Pavlova A, Brondke H, Müsebeck J, et al: Molecular mechanisms underlying hemophilia A phenotype in seven females. J Thromb Haemost 7: 976-982, 2009.

20. Favier R, Lavergne JM, Costa JM, et al: Unbalanced $\mathrm{X}$-chromosome inactivation with a novel FVIII gene mutation resulting in severe hemophilia $\mathrm{A}$ in a female. Blood 96: 4373-4375, 2000.

21. Knobe KE, Sjörin E, Soller MJ, Liljebjörn H and Ljung RC: Female haemophilia A caused by skewed X inactivation. Haemophilia 14: 846-848, 2008.

22. Nair PS, Shetty S and Ghosh K: A homozygous female hemophilia A. Indian J Hum Genet 18: 134-136, 2012.

23. Renault NK, Dyack S, Dobson MJ, et al: Heritable skewed $\mathrm{X}$-chromosome inactivation leads to haemophilia A expression in heterozygous females. Eur J Hum Genet 15: 628-637, 2007.

24. Cai XH, Wang XF, Dai J, et al: Female hemophilia A heterozygous for a de novo frameshift and a novel missense mutation of factor VIII. J Thromb Haemost 4: 1969-1974, 2006.

25. Martín-Salces M, Venceslá A, Alvárez-Román MT, et al: Clinical and genetic findings in five female patients with haemophilia A: Identification of a novel missense mutation, p.Phe2127Ser. Thromb Haemost 104: 718-723, 2010.

26. Venceslá A, Fuentes-Prior $\mathrm{P}$, Baena M, et al: Severe haemophilia $\mathrm{A}$ in a female resulting from an inherited gross deletion and a de novo codon deletion in the F8 gene. Haemophilia 14: 1094-1098, 2008.

27. Jayandharan G, Shaji RV, Baidya S, et al: Identification of factor VIII gene mutation in 101 patients with haemophilia A: mutation analysis by inversion screening and multiplex PCR and CSGE and molecular modeling of 10 novel missense substitutions. Haemophilia 11: 481-491, 2005.

28. Lakich D, Kazazian HH Jr, Antonarakis SE and Gitschier J: Inversions disrupting the factor VIII gene are a common cause for severe haemophilia A. Nat Genet 5: 236-241, 1993.

29. Jacquemin M, De Maeyer M, D'Oiron R, et al: Molecular mechanisms of mild and moderate hemophilia A. J Thromb Haemost 1: 456-463, 2003.

30. Pipe SW: Functional roles of the factor VIII B domain. Haemophilia 15: 1187-1196, 2009.

31. Green PM, Bagnall RD, Waseem NH and Giannelli F: Haemophilia A mutations in the UK: results of screening one-third of the population. Br J Haematol 143: 115-128, 2008.

32. Repessé Y, Slaoui M, Ferrandiz D, et al: Factor VIII (FVIII) gene mutations in 120 patients with hemophilia A: detection of 26 novel mutations and correlation with FVIII inhibitor development. J Thromb Haemost 5: 1469-1476, 2007. 